\title{
Teste de Coordenação Corporal para Crianças (KTK): aplicações e estudos normativos
}

\author{
Body Coordination Test for Children (KTK): applications and normative \\ studies
}

\author{
A.S. Ribeiro, A.C. David, M.M. Barbacena, M.L. Rodrigues, N.M. França
}

ARTIGO DE REVISÃO | REVIEW ARTICLE

\begin{abstract}
RESUMO
Os objetivos da presente revisão foram: i) demonstrar a utilização do Teste de Coordenação Corporal para Crianças (Körperkoordinationstest Für Kinder - KTK) em variadas populações infantis; ii) descrever estudos que desenvolveram padrões de referência do KTK; e iii) averiguar a existência de padrões de referência do KTK para a coordenação motora de crianças brasileiras. A seleção das publicações realizou-se entre os meses de abril e junho (2010) e foram utilizadas as bases de dados Pubmed, Lilacs e www. google.com/scholar. Os resultados indicaram que o KTK é um instrumento de avaliação do desempenho motor coordenativo adequado para ser aplicado em diferentes populações infantis como portadores de síndrome de Down. A maioria dos estudos que construíram valores de referência para suas populações infantis mostrou valores médios inferiores àqueles estabelecidos a partir do grupo de crianças alemãs de onde se originaram os dados de referência do KTK. Tal fato denota a importância da construção de dados normativos em diferentes países, tendo em vista as variadas características de natureza biológica, social, econômica e cultural a serem consideradas. Por fim, observou-se que não existem dados de referência do KTK para crianças brasileiras, porém, é inegável a importância da aplicação do teste para avaliar a coordenação motora global dessas crianças.

Palavras-chave: coordenação motora, crianças, dados normativos, KTK
\end{abstract}

ABSTRACT

The aims of the present review were: i) to demonstrate the use of the Body Coordination Test for Children (Körperkoordinationstest Für Kinder - KTK) in diverse populations of children, ii) to describe studies that developed reference values for KTK, and iii) to ascertain the existence of reference values of KTK for Brazilian children's motor coordination. The selection of publications was performed between April and June (2010) and the Pubmed, Lilacs and www.google.com/scholar databases were employed. The results indicated that the KTK is an evaluation instrument for motor coordinative performance appropriate to be applied in different childish populations, such as Down syndrome bearers. Most studies that constructed reference values for their childish population showed lower mean values than those established from the group of German children from whom originated the reference data of KTK. This fact demonstrates the importance of the construction of normative data in different countries in view of diverse biological, social, economical and cultural characteristics to be considered. Finally, it was observed the absence of reference data of KTK for Brazilian children, however, it is undeniable the importance of applying the test to evaluate the global motor coordination of these children.

Keywords: motor coordination, children, normative data, KTK

Submetido: 01.05.2011 | Aceite: 29.08.2012

Alice Sá Carneiro Ribeiro, Ana Cristina de David, Marcella Manfrin Barbacena e Michele Lopes Rodrigues. Programa de Pós-Graduação Stricto Sensu da Faculdade de Educação Física, Universidade de Brasília, Brasil.

Nanci Maria de França. Programa de Pós-Graduação Stricto Sensu em Educação Física, Universidade Católica de Brasília, Brasil.

Endereço para correspondência: Alice Sá Carneiro Ribeiro, Universidade de Brasília, Faculdade de Educação Física, Via L4 Norte, Setor de Península Norte, 70919-000, Brasília, DF, Brasil.

E-mail: alicesacarneiro@gmail.com 
A coordenação motora, sob uma perspectiva pedagógica e clínica, é a interação harmoniosa e econômica dos sistemas musculoesquelético, nervoso e sensorial para produzir ações cinéticas precisas e equilibradas (Kiphard, 1976). Quanto maior o nível de complexidade de uma tarefa motora, maior o nível de coordenação necessário para um desempenho eficiente (Gallahue \& Ozmun, 2005).

Durante anos foram muitas as denominações utilizadas para a condição de debilidade ou insuficiência no desempenho coordenativo, entre elas: dispraxia, criança com dificuldade motora, disfunção perceptivo-motora, déficits de atenção, de controle motor e de percepção (Santos, Dantas, \& Oliveira, 2004). Kiphard (1977) definiu essa alteração coordenativa como uma instabilidade motora com deficiência qualitativa do movimento associada a uma imperfeição do conjunto da função sensório-motora muscular. De acordo com este autor, a criança apresenta insegurança em postura equilibrada, deficiência da fluidez do movimento, deficiência da precisão da direção, falta de agilidade, dentre outras características. No final da década de 80, a Associação Americana de Psiquiatria (American Psychiatric Association [APA], 2002) reconheceu essa condição, que passou a ser denominada de Transtorno do Desenvolvimento da Coordenação (TDC). Esse transtorno caracteriza-se por um comprometimento do desempenho de atividades diárias, tendo como base a idade cronológica e a inteligência, sem associação com uma condição médica (Santos et al., 2004). Refere-se às crianças com inteligência normal, que não apresentam sinais de lesão cerebral ou de outras doenças conhecidas, mas que exibem coordenação motora abaixo do esperado para a sua idade (APA, 2002).

De acordo com Gorla, Rodrigues, Brunieira, e Guarido (2000) existem diferentes métodos para identificar e avaliar o desempenho motor de crianças, incluindo o seu desempenho coordenativo. Os mais destacados, que avaliam crianças em idades pré-escolar e escolar, segundo os mesmos autores, são: o Movement Assessment Battery for Children (M-ABC), o Teste de Proficiência Motora Bruininks-Oseretsky, o Developmental Test of Visual-Motor Integration (VMI) e o Teste de Coordenação Corporal para Crianças (Körperkoordinationstest Für Kinder - KTK). Este último tem sido utilizado em literaturas nacional e internacional, devido à sua simplicidade e ao seu baixo custo operacional, e será o foco desta revisão.

O Teste de Coordenação Corporal para Crianças (Körperkoordinationstest Für Kinder - KTK), desenvolvido pelos pesquisadores alemães Kiphard e Schilling (1974), foi construído com o propósito de diagnosticar mais sutilmente as deficiências motoras em crianças com lesões cerebrais e/ou desvios comportamentais (Gorla, Araújo, \& Rodrigues, 2009). O teste envolve componentes da coordenação corporal como: o equilíbrio, o ritmo, a força, a lateralidade, a velocidade e a agilidade. Esses componentes foram distribuídos em quatro tarefas que estão contidas em um fator designado por coordenação corporal, identificado por meio de vários estudos empíricos, que utilizaram a análise fatorial exploratória (Gorla et al., 2009).

De acordo com Gorla et al. (2000), o teste foi construído primeiramente para a determinação da situação de desenvolvimento do domínio corporal de crianças portadoras de deficiências. Porém, atualmente observa-se que tem sido utilizado com diversos grupos, inclusive com crianças sem deficiências, já que tanto avalia a coordenação motora global como identifica crianças com distúrbios coordenativos/motores. Nota-se, assim, a necessidade de desenvolver pesquisas direcionadas a obter valores de referências para populações específicas, uma vez que os valores normativos do teste foram reportados há 36 anos e são oriundos de um contexto particular.

Diante do exposto, o presente estudo de 
revisão tem os seguintes objetivos: i) demonstrar a utilização do Teste de Coordenação Corporal para Crianças (Körperkoordinationstest Für Kinder - KTK) em variadas populações infantis; ii) descrever estudos que desenvolveram padrões de referência do KTK; e, iii) averiguar a existência de padrões de referência do KTK para a coordenação motora de crianças brasileiras.

A seleção das publicações foi realizada entre os meses de abril e junho de 2010, sendo utilizadas as bases de dados Pubmed, Lilacs $e$ www.google.com/scholar. Deu-se preferência aos estudos de relevância e que estavam de acordo com os objetivos propostos. Assim, foram selecionados 18 publicações, sendo oito nacionais (brasileiras) e 10 internacionais.

\section{Teste de Coordenação Corporal para Crianças - Körperkoordinationstest Für Kinder" (KTK)}

O Teste de Coordenação Corporal para Crianças (KTK) foi estruturado com 1228 crianças alemães. Pode ser utilizado com crianças entre os cinco anos e os 14 anos e 11 meses e a sua aplicação tem duração de aproximadamente 10 - 15 minutos por criança. Como citado, o teste foi constituído de quatro tarefas: trave de equilíbrio, saltos monopedais, saltos laterais e transferência sobre plataformas. $\mathrm{Na}$ primeira tarefa, verifica-se principalmente $o$ equilíbrio dinâmico; na segunda, a força dos membros inferiores; na terceira, velocidade; e na quarta, lateralidade e estruturação espaço-temporal (Gorla et al., 2009).

Os testes propostos pelo KTK podem ser aplicados individualmente, apresentando confiabilidade de 0.65 a 0.87 , mas ao se realizar a bateria completa, há confiabilidade de 0.90 , o que demonstra credibilidade para a sua aplicação (Gorla et al., 2009).

Para avaliar a capacidade coordenativa utilizando as tabelas originais do estudo de Kiphard e Schilling (1974), é necessário transformar o resultado final de cada tarefa (valores brutos) em quocientes motores (QM). Esse procedi- mento é realizado verificando-se as tabelas de referência para cada teste de acordo com o sexo e a idade do participante para, por fim, realizar o somatório e obter o QM total. Este quociente motor remete a um novo QM, que, por sua vez, permite a classificação da coordenação motora em cinco níveis: muito boa coordenação motora global; boa coordenação motora global; coordenação motora global normal; insuficiência da coordenação motora global; e perturbação na coordenação motora global (Gorla et al., 2009). É importante destacar que, segundo os autores alemães, em uma população saudável, um escore abaixo de 85 representa um desempenho motor abaixo do percentil 15th e um escore inferior a 70, abaixo do percentil 3th. Ambos são considerados preocupantes para a existência de problemas coordenativos (Smits-Engelsmann, Henderson, \& Michels, 1998; Hanewinkel-van Kleef, Helders, Takken, \& Engelbert, 2009; Vandorpe et al., 2010).

\section{Aplicação do KTK em crianças saudáveis}

No Brasil, o estudo de Gorla, Duarte, e Montagner (2008) analisou a coordenação motora, utilizando a bateria do KTK, em 283 escolares, de seis a oito anos, de ambos os sexos, da cidade de Umarama (PR). Realizou-se comparação das pontuações obtidas em cada tarefa (média e desvio-padrão) entre os dados do estudo e do teste original (Kiphard \& Schilling, 1974). Os resultados indicaram diferença significativa nos testes trave de equilíbrio e transferência sobre plataformas em todas as idades. Além disso, também foi observado uma superioridade em quase todos os testes para a população brasileira em relação à alemã nas idades de seis a sete anos e 11 meses, sendo que o mesmo não foi observado aos oito anos e 11 meses. Este fato leva a pensar, segundo os autores, que a população brasileira seria mais ativa que a alemã, da época de padronização do teste original, na primeira faixa etária. Em relação ao sexo, ocorreu diferença significativa apenas aos sete anos, na tarefa saltos mono- 
pedais, em favor dos meninos, o que pode ser, provavelmente, justificado pela maior resistência de força, segundo os autores.

O estudo de Smits-Engelsmann, Henderson, e Michels (1998) destacou a importância da utilização do KTK como instrumento de avaliação da coordenação motora em crianças holandesas. Este foi realizado com 208 crianças de ambos os sexos (cinco a 13 anos), para comparar a coordenação motora, de acordo com as médias adquiridas na bateria completa do KTK e no M-ABC ${ }^{1}$. Os resultados indicaram $29 \%$ das crianças com dificuldades coordenativas no KTK e $16 \%$, no M-ABC. Os autores concluíram que as normas do primeiro teste necessitavam de ajustes para a população em estudo, ao contrário do segundo, à medida que os pontos de corte adotados parecem superestimar o número de crianças holandesas com dificuldades coordenativas.

A relação entre atividade física e coordenação motora também foi objeto de estudo de Lopes, Rodrigues, Maia, e Malina (2009), que acompanharam 285 crianças da Região dos Açores, dos seis aos 10 anos. Os autores propuseram avaliar a coordenação motora das crianças por meio da bateria completa do KTK e observar se esta competência pode ser considerada preditora da atividade física. Os resultados indicaram uma diminuição geral da atividade física ao longo dos três anos de acompanhamento, mas essa tendência pode ser atenuada ou aumentada, a depender do nível inicial de coordenação motora da criança. Assim, aquelas crianças que apresentaram níveis mais baixos de coordenação motora aos seis anos demonstraram um maior declínio dos níveis de atividade física no intervalo de tempo considerado pelo estudo, seguidas daquelas com níveis iniciais médios de coordenação motora. $\mathrm{E}$ as crianças com níveis altos aos seis anos apre1 - M-ABC (Movement Assessment Battery for Children) avalia a coordenação motora global e a fina. Foi desenvolvido por Herderson e Sugden (1982) e normatizado com 1234 crianças americanas podendo ser aplicado com crianças entre quatro e 12 anos. sentaram alterações imperceptíveis da atividade física ao longo do tempo etário analisado. $\mathrm{Ou}$ seja, a coordenação motora mensurada pelo KTK pode ser considerada como preditora significante da atividade física em crianças de seis anos avaliadas até os 10 anos de idade. Dessa forma, segundo os autores, a partir da perspectiva da saúde pública, investir na proficiência motora de crianças pequenas tem implicações políticas potencialmente relevantes, relacionadas com a atividade física por si só e com os benefícios para a saúde.

\section{Estudos com crianças portadoras de deficiência sensorial}

Gorla, Araújo, e Carminato (2004) analisaram o progresso de crianças portadoras de deficiência intelectual após um programa de prática sequencial com duração de 10 semanas, totalizando 23 sessões. Participaram nove sujeitos com idade entre seis e 11 anos, em Rolândia (PR). Aplicou-se a tarefa saltos monopedais e verificou-se evolução significativa no grupo como um todo, após a intervenção realizada, apesar de dois dos participantes não terem apresentado desempenho satisfatório no pós-teste. Isso pode ter ocorrido, segundo os autores, por diversidade das dificuldades individuais como, por exemplo, déficit de força de membros inferiores, demonstrado por dificuldade na permanência do apoio unipodal. Apesar de ser considerada de fácil aplicação, essa tarefa talvez não seja adequada para ser aplicada em crianças com deficiência intelectual, de acordo com os autores.

O estudo de caso de Lima e Almeida (2008) avaliou a influência do aprendizado da natação (24 aulas) na coordenação motora de uma criança, de Uberlândia(MG), deficiente visual congênita de 10 anos. Aplicaram-se duas tarefas do KTK: trave de equilíbrio e saltos laterais. As citadas tarefas foram selecionadas, visto que as tarefas saltos monopedais e transferência sobre plataformas exigem controle visual em sua execução, sendo suas aplicações inade- 
quadas para crianças com deficiência visual. Nesse estudo, também foi utilizado um período de linha de base para observar os comportamentos motores apresentados pela criança dentro da piscina. Os resultados referentes ao equilíbrio dinâmico e a velocidade em saltos laterais mostraram um pequeno ganho quantitativo nas duas tarefas (um ponto no quociente motor final da primeira tarefa e dois pontos no da segunda tarefa).

Por sua vez, Souza, Gorla, Araújo, Lifante, e Campana (2008) avaliaram a coordenação motora de 23 crianças com deficiência auditiva (seis com deficiência unilateral e $17 \mathrm{com}$ deficiência bilateral), de sete a 14 anos (13 meninos e 10 meninas), de Campinas (SP). Foi aplicada a bateria completa do KTK, antes e depois de um programa de intervenção de 14 sessões de atividades específicas. Na realização do pré-teste, o KTK foi utilizado para identificar as variáveis em que os participantes apresentavam maior dificuldade, a fim de direcionar as atividades do programa de intervenção. Os resultados indicaram progresso no desempenho coordenativo total após o programa proposto. Porém, para ambos os sexos, observou-se diminuição do rendimento na tarefa trave de equilíbrio, apesar de não ter sido observada diferença significativa. Esse resultado pode ter ocorrido, segundo os autores, porque os participantes apresentaram alguns aspectos individuais e particulares do deficiente auditivo tais como: dificuldade de concentração, déficit de atenção durante a execução das tarefas, impaciência, privação de vivência e de experiência nas atividades propostas. Contudo, ao serem analisadas as outras tarefas, houve aumento significativo do rendimento motor do pós-teste em relação ao pré-teste em ambos os sexos, com exceção da tarefa transferência sobre plataformas, no sexo feminino. Ao considerar a tarefa salto monopedais isoladamente, observou-se que no pós-teste a média foi significativamente maior nas duas pernas $(p<.001-$ perna direita; $p<$ .05 - perna esquerda), no sexo masculino, e na perna direita $(p<.05)$, no sexo feminino.

\section{Estudos com crianças com características peculiares}

Silva e Ferreira (2001) aplicaram o KTK com nove crianças portadoras de síndrome de Down (quatro meninas e cinco meninos), de seis a 10 anos de idade, da cidade de Maringá (PR). As crianças participaram de um programa de atividades sistematizadas, sendo realizados pré e pós testes. Aplicou-se a bateria completa do KTK para análise da coordenação motora das crianças e observou-se que, de forma geral, houve um aumento significativo do desempenho coordenativo das crianças. Ao isolar as tarefas, observou-se que apenas na tarefa trave de equilíbrio não foi verificado ganho significativo do desempenho motor. Os autores ainda realizaram a classificação da coordenação segundo Kiphard e Schilling (1974) e observaram que as crianças em estudo se apresentaram como de nível baixo, porém, segundo os resultados, pode-se observar que houve melhora na qualidade das respostas motoras das crianças portadoras de síndrome de Down, à medida que os escores obtidos foram maiores ao final do estudo (pós-teste).

O estudo alemão de Hebestreit, Schrank, Schrod, Strassburg, e Kriemler (2003) verificou, em um dos seus objetivos, a relação entre o perímetro cefálico e o desempenho motor coordenativo de crianças nascidas prematuras e de baixo peso ( 21 meninas e 12 meninos), entre seis e 12 anos. Utilizou-se a bateria completa do KTK neste estudo. O perímetro cefálico foi mensurado no dia da avaliação coordenativa e os resultados não indicaram diferença entre a coordenação motora de crianças prematuras que apresentaram perímetro cefálico pequeno e aquelas que apresentaram dimensão ideal desta variável. Por outro lado, ao considerar as crianças prematuras com perímetro cefálico pequeno e ideal como apenas um grupo e compará-lo com o grupo controle de crianças a termo, observou-se pior resultado para o 
primeiro grupo $(p<.05)$. Concluiu-se nesse estudo que, de acordo com o instrumento aplicado, os prematuros apresentaram desempenho coordenativo inferior ao de crianças nascidas a termo.

O desempenho motor de crianças holandesas portadoras de hipermobilidade articular generalizada foi analisado por um estudo recente (Hanewinkel-van Kleef et al., 2009), aplicando a bateria completa do KTK e o M-ABC. O objetivo foi verificar se a força muscular e a capacidade funcional ao exercício de 41 crianças, de cinco a 12 anos, influenciavam o seu desempenho motor. Os resultados indicaram que a força muscular pode ser considerada preditora do desempenho coordenativo, quando utilizado o KTK. No entanto, não houve associação do KTK com a capacidade funcional ao exercício. De acordo com os critérios do M-ABC, 32 crianças $(78 \%)$ apresentavam desempenho normal, três crianças (7.4\%) apresentaram risco de déficit motor e seis crianças (14.6\%) apresentaram atraso definitivo no desempenho motor. De acordo com a classificação do KTK, nove crianças $(22 \%)$ apresentaram desempenho normal, 15 (36.5\%) apresentaram risco de déficit motor e 17 (41.5\%) atraso motor definitivo. Nota-se que o KTK mostrou-se mais sensível na detecção de problemas motores de crianças com hipermobilidade articular generalizada que o M-ABC.

\section{Estudos avaliando a influência do sobrepeso/ obesidade na coordenação motora}

No estudo de Catenassi et al. (2007), onde participaram 27 crianças com idade média de $5.6 \pm 0.7$ (16 meninos e 11 meninas), de Londrina (PR), foi verificado o índice de massa corporal (IMC) e selecionados dois instrumentos para avaliar o desenvolvimento motor das crianças: Test of Gross Motor Development - Second Edition (TGMD-2) e o Körperkoordinationstest für Kinder (KTK). Na análise descritiva não se observou diferença entre os gêneros para massa corporal, estatura e IMC, sendo que cinco crianças (18\%) foram classificadas como obesas e quatro (15\%), como sobrepesos. Porém, ao correlacionar o desempenho geral das crianças, nos dois testes propostos, com o IMC, foi verificada baixa correlação tanto para o TGMD-2 $\left(r_{s}\right.$ $=-$.09) quanto para o KTK $\left(r_{s}=-.04\right)$, considerando a pontuação total alcançada por todas as crianças nos testes. Assim, concluiu-se que o desempenho das crianças, analisadas em tarefas que envolviam habilidade motora grossa propostas pelo KTK, não se relacionou com o IMC. Nesse estudo, os autores optaram por não utilizar as tabelas de referência do teste original para obter a variável final coordenação motora. Esta variável foi estudada considerando o somatório das pontuações obtidas nos quatro testes que compõem o KTK.

Outro estudo brasileiro (Collet, Folle, Pelozin, Botti, \& Nascimento, 2008), de Florianópolis (SC), aplicou a bateria do KTK em 243 escolares (8 a 14 anos), considerando sexo, faixa etária, prática extraescolar e IMC. Os autores adaptaram os pontos de corte do QM total por meio da redistribuição das frequências por tercis: nível baixo ( $1^{\circ}$ tercil: $65<\mathrm{QM}$ $<106)$, nível normal $\left(2^{\circ}\right.$ tercil: $107<\mathrm{QM}<$ $118)$ e nível alto ( $3^{\circ}$ tercil: $119<\mathrm{QM}<140$ ). Os resultados revelaram que os meninos apresentaram níveis mais elevados de coordenação motora (17.6\%, 32.4\%, 50\% - níveis baixo, normal e alto, respectivamente; $p<.001$ ), do que as meninas $(49.6 \%, 32.6 \%, 17.8 \%$ - níveis baixo, normal e alto, respectivamente). Estas apresentaram percentual expressivo de baixa coordenação. Observou-se um decréscimo significativo $(p=.023)$ nos níveis de coordenação nas crianças com idade mais avançada. Enquanto as crianças de oito a nove anos apresentaram os maiores níveis de coordenação motora, aquelas com 12 a 14 anos revelaram índices expressivos de baixa coordenação para esta faixa etária. Este fato diverge dos resultados encontrados em outros estudos que relatam níveis maiores de coordenação motora à medida que a idade avança (Kiphard \& Schi- 
lling, 1974; Lopes, Maia, Silva, Seabra, \& Morais, 2003; Valdivia et al., 2008a; Valdivia et al., 2008b; Vandorpe et al., 2010). Em relação à prática de esportes, apesar de não ter sido encontrada diferença significativa, a maioria das crianças que praticava esportes apresentou alta coordenação em comparação com aquelas não-praticantes que, por sua vez, apresentaram índices similares de baixa coordenação e de coordenação normal. No que se refere ao IMC, a maioria das crianças com sobrepeso/obesidade $(54.7 \%)$ apresentou nível baixo de coordenação. Pode-se concluir, de acordo com os achados desse estudo, que o sexo, a faixa etária, a prática de atividades esportivas extraescolar e o IMC influenciaram o nível de coordenação motora de crianças e jovens em idade escolar ao serem avaliados pelo KTK.

O estudo de Graf et al. (2004) visou correlacionar o IMC, os hábitos de lazer e as habilidades motoras de 668 crianças alemães de seis e sete anos ( 351 meninos e 317 meninas), aplicando o KTK e o teste de corrida de seis minutos. As crianças foram classificadas em $5.7 \%$ obesas, $8.1 \%$ sobrepeso, $78.1 \%$ eutróficas e $8.1 \%$ abaixo do peso. Os meninos apresentaram resultados significativamente melhores que as meninas $(p<.001)$. Os resultados dos dois testes foram inversamente correlacionados com o IMC $\left(r_{s}=-.162\right.$ e $r_{s}=-.209$, respectivamente, para o KTK e para o teste de corrida de seis minutos; $p<.001)$. As crianças do grupo sobrepeso/obeso apresentaram resultados inferiores ao do grupo de crianças eutróficas, mesmo quando ajustados pelo gênero e pela idade. Apesar de ser uma correlação fraca, os resultados indicaram que o alto índice de gordura corporal parece interferir negativamente no desempenho da coordenação motora e da resistência. Os autores salientaram ainda que um estilo de vida mais ativo está possivelmente correlacionado com um melhor desenvolvimento de habilidades globais no primeiro grau do ensino escolar.

Outro estudo (Graf et al., 2005) avaliou a influência de um programa de prevenção da obesidade (atividade física diária, nutrição e aulas educativas), de aproximadamente 20 meses de duração, no IMC e nas habilidades motoras de 651 crianças $(6.85 \pm 0.47$ anos). Para avaliar o desempenho motor, foi utilizada a tarefa saltos laterais da bateria do KTK, além de se comparar os resultados com crianças de um grupo controle. Os resultados indicaram que todas as crianças dos dois grupos estudados (controle e intervenção) apresentaram aumento do número de saltos laterais no decorrer do estudo $(p<.001)$, mas o aumento foi maior no grupo intervenção (6.5 pulos a mais em média). Segundo a classificação do IMC (obesidade, sobrepeso, eutrófico, baixo peso), as crianças eutróficas do grupo intervenção apresentaram aumento significativo após o programa realizado quando comparadas àquelas do grupo controle. As crianças classificadas como sobrepeso ou obesidade apresentaram valores inferiores nesta tarefa, tanto antes como depois do programa de intervenção, sem, portanto, haver diferença significativa entre as crianças do grupo intervenção e controle, antes e após o programa.

O estudo de Valdivia et al. (2008a) visou determinar a influência da idade, do sexo, do nível socioeconômico e do nível de adiposidade subcutânea (dobras cutâneas do tríceps, subescapular e panturrilha) na coordenação motora de 4007 escolares peruanos (seis a 11 anos). Houve aumento dos valores médios nas quatro tarefas do KTK ao longo das idades e os autores destacaram maior variabilidade em torno dos valores médios nas tarefas saltos laterais e transferência sobre plataformas, em ambos os sexos. Os meninos apresentaram melhores resultados que as meninas nas tarefas saltos monopedais e transferência sobre plataformas $(p<.001)$. Porém, as meninas foram melhores que os meninos na tarefa trave de equilíbrio $(p<.001) . \mathrm{Na}$ tarefa saltos laterais não houve diferença significativa entre os sexos. Nesse estudo, para verificar a coordenação motora 
global, os autores não consideraram os pontos de corte e as tabelas normativas do teste original. Por outro lado, optou-se por realizar o somatório das pontuações obtidas nas quatro tarefas propostas pelo KTK, assim como realizado por Catenassi et al. (2007). A partir deste procedimento, observou-se que os meninos apresentaram coordenação motora melhor que as meninas em todas as idades analisadas. Este processo parece indicar que a popularidade do KTK faz tornar cuidadosa a consideração dos pontos de corte, baseados na amostra alemã. Em relação ao nível socioeconômico, os autores destacaram que este não parece ser um preditor conclusivo na coordenação motora. E finalmente, aquelas crianças com adiposidade classificada como média e alta apresentaram desempenho coordenativo inferior ao demonstrado por crianças com adiposidade baixa, em ambos os sexos. Fato que corrobora os estudos referentes ao desempenho motor de crianças com sobrepeso (Graf et al., 2004; Graf et al., 2005).

Esse estudo também remete a uma abordagem comparativa entre as amostras portuguesa de 2007 (Maia \& Lopes, 2007) e alemã de 1974 (Kiphard \& Schilling, 1974). Observou-se uma superioridade dos resultados para esta última em todas as tarefas do KTK, principalmente na tarefa transferência sobre plataformas, em que a diferença foi mais que a metade entre os valores médios apresentados pelas crianças alemães em relação aos seus pares peruanos e portugueses. Ao examinar os dados peruanos e portugueses no teste trave de equilíbrio, observou-se uma moderada superioridade em ambos os sexos dos escolares peruanos. Na tarefa saltos laterais, constatou-se que os melhores desempenhos foram observados nas crianças portuguesas. Nos saltos monopedais, apenas distinguiu-se diferença no sexo masculino, enquanto que, na tarefa transferência sobre plataformas, os valores foram semelhantes em ambos os sexos. E com esse estudo peruano, pôde-se verificar a influência de fatores ambientais, de estilo de vida, de padrão alimentar e de nível socioeconômico no desenvolvimento da coordenação motora.

Estudos para construção de dados normativos da bateria do KTK em diferentes países

Os estudos que propuseram estabelecer valores de referência de seus países, visando facilitar um diagnóstico e um perfil de desempenho coordenativo, segundo os testes do KTK, mais adequados para as suas realidades (Valdivia et al., 2008a; Valdivia et al., 2008b; Vidal et al., 2009; Vandorpe et al., 2010).

No Peru, o estudo de Valdivia et al. (2008b), com os mesmos participantes do estudo anteriormente citado (Valdivia et al., 2008a), realizou uma distribuição de percentis $(\mathrm{P})$ do desempenho coordenativo de 4007 escolares da área metropolitana de Lima. Por meio do modelo matemático/estatístico LMS, relatou-se os gráficos de referências para todos os testes considerando os percentis $3,10,25,50,75,90$ e 95, aceitáveis para descrever a forte variação existente em cada tarefa. Os percentis, segundo Vidal et al. (2009), podem auxiliar, ainda que sem fundamentação clínica e/ou pedagógica, na determinação do desempenho coordenativo, considerando que os valores abaixo do P10 expressam um desempenho coordenativo insuficiente, valores entre os P10 e P90 são considerados adequados para uma dada idade e sexo, e valores acima do $\mathrm{P} 90$ são considerados superiores. Houve um padrão de desenvolvimento coordenativo específico de cada sexo e se observou que a pontuação das meninas no P50 variou de 32 a 50 na tarefa trave de equilíbrio, de 17 a 45 na tarefa saltos monopedais, de 24 a 49 na tarefa saltos laterais e de 12 a 20 na tarefa transferência sobre plataformas. Em relação aos meninos, as pontuações no mesmo percentil foram (respectivamente nas tarefas citadas) de 28 a 50 , de 21 a 54 , de 25 a 50 e de 13 a 21 . Assim, os autores destacam que os meninos apresentaram valores médios significativamente melhores que as meninas nas tarefas saltos monopedais e transferência 
sobre plataformas em todas as idades. Característica semelhante ocorreu na tarefa saltos laterais com exceção dos 10 anos de idade, em que as meninas se sobressaíram, porém sem diferença significativa. No entanto, na tarefa trave de equilíbrio, as meninas apresentaram melhor desempenho coordenativo. Os dados indicam também que $65.26 \%$ das meninas e $68.28 \%$ dos meninos, aos seis anos, estavam bem classificados em relação ao seu desempenho coordenativo. Porém, a partir dos sete anos houve uma tendência ao decréscimo desse desempenho até os 10 anos, em ambos os sexos, indicando número reduzido de crianças com desempenho coordenativo satisfatório nessas idades. Ao comparar os dados desse estudo com o original do KTK, na faixa etária entre seis e 11 anos, verificaram-se valores médios inferiores para a amostra peruana em todos os testes motores.

Em Portugal, o estudo de Vidal et al. (2009), para construir dados de referência para a população infantil deste país, agrupou dados de 4724 crianças (seis a 11 anos), provenientes de dois estudos da Região de Açores, um de caráter transversal (Lopes et al., 2003) e outro longitudinal (Maia \& Lopes, 2007), em que as crianças foram acompanhadas por um período de quatro anos. Os autores não recorreram aos dados normativos alemães e consideraram o desempenho da criança em cada prova (valores brutos), não obtendo, assim, os QMs referenciados segundo os autores do KTK. Da mesma forma como o estudo anterior (Valdivia et al., 2008b), os autores utilizaram os percentis que são importantes para descrição e diagnóstico. Ao comparar os resultados açorianos com os peruanos (Valdivia et al., 2008b), os autores observaram desempenho coordenativo inferiores, no P50, nas tarefas trave de equilíbrio e saltos monopedais, variando de 22.52 a 45.66 (trave de equilíbrio), 14.43 a 45.32 (saltos monopedais), nos meninos; e 25.25 a 45.64 (trave de equilíbrio) e 11.78 a 45.62 (saltos monopedais), nas meninas. Porém, na tarefa saltos laterais as crianças açorianas foram melhores que as peruanas (variação de 29.29 a 54.24 , nos meninos, e de 27.18 a 54.16 , nas meninas). Na tarefa transferência sobre plataformas se observaram diferenças em cada uma das idades, em ambos os sexos. Segundo os autores, uma das características essenciais desse estudo foi a capacidade de auxiliar na interpretação do desempenho coordenativo de crianças açorianas, em cada teste do KTK. Além disso, pode-se verificar que, em todas as faixas etárias estudadas, os valores médios obtidos pela amostra portuguesa foram inferiores àquelas apresentadas pela amostra original do teste. Resultado que se assemelha ao encontrado por Valdivia et al. (2008b).

$\mathrm{O}$ estudo mais recente visando construir valores de referências atualizados em relação ao sexo e a idade, foi desenvolvido na Bélgica, Região de Flandres, com 2470 crianças entre 6 e 12 anos (Vandorpe et al., 2010). Segundo os autores, os meninos apresentaram melhor desempenho coordenativo que as meninas, embora ambos os sexos tenham apresentado escores inferiores aos da amostra alemã $(p<$ .001). Ao comparar, de forma geral, as crianças da região de Flandres com as crianças alemães, as primeiras apresentaram resultados inferiores nas tarefas trave de equilíbrio e transferência sobre plataformas superando as segundas nas tarefas saltos monopedais e saltos laterais. Nesses últimos testes, os meninos da região de Flandres apresentaram desempenho coordenativo melhor que os da amostra alemã. Enquanto que as meninas das amostras analisadas apresentaram desempenhos semelhantes considerando que, em algumas idades, as meninas da região de Flandres foram melhores que as alemães e em outras, piores. Ao verificar a distribuição da classificação das crianças das duas amostras em estudo, observou-se $21.1 \%$ das crianças da região de Flandres com distúrbios motores em comparação com $16 \%$ da amostra alemã $(p<.001)$. Enquanto que $8.7 \%$ das crianças da região de Flandres apresentaram coordenação "boa" e "muito boa", 
o índice dentre as alemães era de $16 \%$. Esse resultado mostra que a distribuição de crianças belgas em 2008 desviou daquela apresentada por crianças alemães em 1974, principalmente nos resultados dos melhores níveis de coordenação. Apesar disso, os autores do estudo revelaram que não existe razão para ajustar os pontos de corte para a população da região dos Flandres.

No Brasil, as publicações analisadas indicaram a inexistência de padrões de referência dos testes do KTK para sua população infantil. Apenas o estudo de Gorla et al. (2009) propôs, por meio de um modelo matemático, uma equação de regressão linear múltipla que funciona como parâmetro de análise da coordenação motora global de portadores de deficiência intelectual. Para isso, utilizou-se dados de 160 crianças (80 meninas e 80 meninos) apresentando idades entre sete e 14 anos. Os autores propuseram uma matriz de reclassificação da coordenação motora global tanto para o gênero masculino como para o feminino.

\section{Considerações Finais}

Os estudos relatados indicam que a bateria do KTK é um instrumento de avaliação do desempenho motor coordenativo adequado para ser aplicado em diferentes populações como crianças saudáveis, crianças portadoras de deficiência sensorial ou que apresentam características peculiares como síndrome de Down, hipermobilidade articular e aquelas nascidas prematuras, bem como aquelas crianças que apresentam sobrepeso/obesidade.

A maior parte dos resultados descritos nos estudos, que construíram valores de referência para sua população, mostrou valores médios inferiores aqueles estabelecidos pelas crianças alemãs que fizeram parte do grupo de onde foi originado os dados de referência do KTK. Tal fato indica a importância de dados que possam ser comparados aos dados referenciais do teste KTK em diferentes países, tendo em vista as variadas características de natureza biológica, social, econômica e cultural a serem consideradas.

No Brasil, existem dados de referência da bateria KTK para crianças portadoras de deficiência intelectual de sete a 14 anos. Porém, ainda não há uma validação do teste para crianças brasileiras em geral. Apesar disso, é inegável a importância do teste, pois este permite detectar, com margem de erro muito pequena, as diferenças na coordenação motora grossa, tanto em populações saudáveis como naquelas que apresentam perturbações no desenvolvimento (Gorla et al., 2009). Assim, nota-se a necessidade da realização de estudos que estabeleçam dados de referência para o desempenho coordenativo de crianças dentro da realidade brasileira, como tem sido desenvolvido em outros países, a fim de se obter pontos de corte adequados para possibilitar comparações entre diferentes populações. Além do mais, como citado por Vidal et al. (2009), esse processo permitiria especificar valores de referência, traçar perfis multidimensionais das crianças e posicioná-las em termos relativos a uma população estudada de acordo com a idade e o sexo.

\section{Agradecimentos:}

Nada declarado.

\section{Conflito de Interesses:}

Nada declarado.

Financiamento:

Nada declarado.

\section{REFERÊNCIAS}

American Psychiatric Association. (2002). Manual diagnóstico e estatístico de transtornos mentais: DSM-IV-TR. Porto Alegre: Artmed.

Catenassi, F. Z., Marques, I., Bastos, C. B., Basso, L., Ronque, E. R. V., \& Gerage, A. M. (2007). Relação entre índice de massa corporal e habilidade motora grossa em crianças de quatro a 
seis anos. Revista Brasileira de Medicina do Esporte, $13(4), 227-230$.

Collet, C., Folle, A., Pelozin, F., Botti, M., \& Nascimento, J. V. (2008). Nível de coordenação motora de escolares da rede estadual da cidade de Florianópolis. Motriz, 14(4), 373-380.

Gallahue, D. L., \& Ozmun, J. C. (2005). Compreendendo o desenvolvimento motor: bebês, crianças, adolescentes e adultos (3 ed.). São Paulo: Phorte.

Gorla, J. I., Araújo, P. F., \& Carminato, R. A. (2004). Desempenho psicomotor em portadores de deficiência mental: avaliação e intervenção. Revista Brasileira de Ciência do Esporte 25(3), 133-147.

Gorla, J. I., Araújo, P. F., \& Rodrigues, J. L. (2009). Avaliação motora em educação física adaptada. São Paulo: Phorte.

Gorla, J. I., Duarte, E., \& Montagner, P. C. (2008). Avaliação da coordenação motora de escolares da área urbana do Município de Umuarama-PR Brasil. Revista Brasileira de Ciência e Movimento, 16(2), 57-65.

Gorla, J. I., Rodrigues, J. L., Brunieira, C. A. V., \& Guarido, E. A. (2000). Teste de avaliação para pessoas com deficiência mental: identificando o KTK. Arquivos de Ciência da Saúde da Unipar, 4(2), 121-128.

Graf, C., Koch, B., Falkowski, G., Jouck, S., Christ, H., Stauenmaier, K.,...Predel, H. (2005). Effects of a school-based intervention on BMI and motor abilities in childhood. Journal of Sports Science and Medicine, 4, 291-299.

Graf, C., Koch, B., Kretschmann-Kandel, E., Falkowski, G., Christ, H., Coburger, S.,... Dordel, S. (2004). Correlation between BMI, leisure habits and motor abilities in childhood (CHILT-Project). International Journal of Obesity, 28(1), 22-26.

Hanewinkel-van Kleef, Y. B., Helders, P. J., Takken, T., \& Engelbert, R. H. (2009). Motor performance in children with generalized hypermobility: the influence of muscle strength and exercise capacity. Pediatric Physical Therapy, 21 (2), 194-200.

Hebestreit, H., Schrank, W., Schrod, L., Strassburg, H. M., \& Kriemler, S. (2003). Head size and motor performance in children born prematu- rely. Medicne \& Science in Sports \& Exercise, 35(6), 914-922.

Kiphard, E. J. (1976). Insuficiências de movimiento y de coordinación en la edad de la escuela primaria. Buenos Aires: Kapeluscz.

Kiphard, E. J. (1977). Bewugungs-und Koordination-Schwächem in Grudschulater. Hofmann Verlag: Schondorf.

Kiphard, E. J., \& Schilling, V. F. (1974). Körper-koordinations-test für kinder KTK: manual Von Fridhelm Schilling. Weinhein: Beltz Test.

Lima, S. R., \& Almeida, M. A. (2008). Iniciação à aprendizagem da natação e a coordenação corporal de uma criança deficiente visual: algumas contribuições. Revista Brasileira de Ciência do Esporte, Campinas, 29(2), 57-78.

Lopes, V. P., Maia, J. A. R., Silva, R. G., Seabra, A., \& Morais, F. P. (2003). Estudo do Nível de Desenvolvimento da Coordenação Motora da População Escolar (6 a 10 Anos de Idade) da Região Autónoma dos Açores. Revista Portuguesa de Ciências do Desporto 3(1), 47-60.

Lopes, V. P., Rodrigues, L. P., Maia, J. A., \& Malina, R. M. (2009). Motor coordination as predictor of physical activity in childhood. Scandinavian Journal of Medicine \& Science in Sports, 1-7.

Maia, J. A., \& Lopes, V. P. (2007). Crescimento e desenvolvimento de crianças e jovens Açorianos. O que os pais, professores, pediatras e nutricionistas gostariam de saber. Universidade do Porto.

Santos, S., Dantas, L., \& Oliveira, J. A. (2004). Desenvolvimento motor de crianças, de idosos e de pessoas com transtornos da coordenação. Revista Paulista de Educação Física, 18, 33-44.

Silva, D. R., \& Ferreira, J. S. (2001). Intervenção na educação física em crianças com Síndrome de Down. Revista da Educação Física/UEM, 12(1), 69-76.

Smits-Engelsmann, B. C. M., Henderson, S. E., \& Michels, C. G. J. (1998). The assessment of children with Developmental Coordination Disorders in the Netherlands: The relationship between the Movement Assessment Battery for Children and the Körperkoordinations Test 
für Kinder. Human Movement Science, 17(4-5), 699-709.

Souza, A. N., Gorla, J. I., Araújo, P. F., Lifante, S. M., \& Campana, M. B. (2008). Análise da coordenação motora de pessoas surdas. Arquivos de Ciência da Saúde da Unipar, 12(3), 205-211.

Valdivia, A. B., Cartagena, L. C., Sarria, N. E., Távara, I. S., Seabra, A. F. T., Silva, R. M. G., \& Maia, J. A. R. (2008a). Coordinación motora: influencia de la edad, sexo, estatus socioeconômico y niveles de adiposidad en niños peruanos. Revista Brasileira de Cineantropometria \& Desempenho Humano, 10(1), 25-34.

Valdivia, A. B., Lara, R. F., Espinoza, C. B., Pomahuacre, S. Q., Ramos, G. R., Seabra, A., Maia, J. (2008b). Prontitud coordinativa: perfiles multivariados en función de la edad, sexo y estatus socioeconômico. Revista Portuguesa de Ciências do Desporto, 8(1), 34-46.
Vandorpe, B., Vandendriessche, J., Lefèvre, J., Pion, J., Vaeyens, R., Matthys, S.,...Lenoir, M. (2010). The Körperkoordinations Test für Kinder: reference values and suitability for 6-12 years old children in Flanders. Scandinavian Journal of Medicine \& Science \& Sports, 21 (3), 378-388.

Vidal, S. M., Bustamante, A., Lopes, V. P., Seabra, A., Silva, R. G., \& Maia, J. A. (2009). Construção de cartas centílicas da coordenação motora de crianças dos 6 aos 11 anos da Região Autónoma dos Açores, Portugal. Revista Portuguesa de Ciência do Desporto, 9(1), 24-35.

(cc)BY-NC Todo o conteúdo da revista Motricidade está licenciado sob a Creative Commons, exceto quando especificado em contrário e nos conteúdos retirados de outras fontes bibliográficas. 